\title{
HEATER-INDUCED ALTITUDE DESCENT OF THE EISCAT UHF ION LINE ENHANCEMENTS: OBSERVATIONS AND MODELLING.
}

\author{
Mina Ashrafi ${ }^{\text {a,* }}$, M. J. Kosch ${ }^{\mathrm{a}, \mathrm{b}, \mathrm{c}}$, F. Honary ${ }^{\mathrm{a}}$ \\ ${ }^{a}$ Dept. of Communications Systems, Lancaster University, LA1 4WA, UK \\ ${ }^{\mathrm{b}}$ Currently at Air Force Research Laboratory, Hanscom AFB, Boston, USA \\ ${ }^{\mathrm{c}}$ Honorary Research Fellow, University of Kwazulu-Natal, Durban, South Africa
}

\begin{abstract}
On 12 November 2001, artificial optical annuli were produced using the EISCAT highfrequency (HF) ionospheric Heating facility. This unusual phenomenon was induced using O-mode transmissions at $5.423 \mathrm{MHz}$ with $550 \mathrm{MW}$ effective isotropic radiated power and the pump beam dipped 9 degrees south. The pump frequency corresponds to the fourth electron gyro-harmonic frequency at $215 \mathrm{~km}$ altitude. The EISCAT UHF radar observed a persistent pump-induced enhancement in the ion line data near the HF reflection altitude. The optical and radar signatures of HF pumping started at $\sim 230 \mathrm{~km}$ and descended to $\sim 220$ $\mathrm{km}$ within $\sim 60 \mathrm{~s}$. This effect has been modelled using the solution to differential equations describing pump-induced electron temperature and density perturbations. The decrease in altitude of the ion line by $\sim 10 \mathrm{~km}$ and changes in electron density have been modelled. The results show that an electron temperature enhancement of up to $\sim 5700 \mathrm{~K}$ can be achieved, which is not sufficient to explain the observed optical emissions.
\end{abstract}

Key words: Ionospheric heating; Electron acceleration

\section{Introduction}

Large scale electron density and temperature modification as a result of ionospheric heating by high power high frequency (HF) radio waves in the F-region has been studied from the early 1970s. Any bulk modification which occurs on the scale of $10 \mathrm{~km}$ or more perpendicular to the geomagnetic field direction is known as

* Corresponding author. Fax: +44-1524-510493.

Email address: m.ashrafi@lancaster.ac.uk (Mina Ashrafi). 
large scale features in the heated plasma. Electron temperature enhancements have been observed during $\mathrm{O}$-mode heating and measured by incoherent scatter radar at Arecibo, i. e. low latitude, by Gordon et al. (1971) and Gordon and Carlson (1974). At high latitudes the first observations were performed in Troms $\varnothing$, Norway (Jones et al., 1982, 1986; Robinson, 1989; Honary et al., 1993). Peak electron temperature enhancements up to 50\% were measured by Robinson (1989) and by Stocker et al. (1992) in the daytime. More recent observations show that much greater electron temperature enhancements up to $300 \%$ occur at night and when heating along the magnetic field aligned direction (Rietveld et al., 2003). Modelling of electron temperature enhancements by ionospheric heating has been done for both high and low latitudes (Stocker et al., 1992; Mingaleva and Mingalev, 1997; Sergienko et al., 2000; Vas'kov et al., 1993). Using numerical models of the high latitude ionosphere, Mingaleva and Mingalev (1997) found the electron temperature at its peak can increase up to a maximum of $7300 \mathrm{~K}$.

High power HF electromagnetic radio waves can couple to high and low frequency electrostatic plasma waves through parametric instabilities near the reflection height of the pump wave. At the reflection level of the O-mode pump wave the group velocity of the wave decreases to zero and, as a result, the wave electric field maximises, whilst becoming parallel to the direction of the ambient magnetic field line. Due to this field orientation in the so called plasma resonance region, where the plasma frequency equals the wave frequency, the electromagnetic wave can couple to variety of electrostatic waves through various instabilities such as the Oscillating Two Stream Instability (OTSI) and Parametric Decay Instability (PDI) (Rietveld et al., 1993; Kohl et al., 1993). However, only those electrostatic waves with a wave number half that of the probing radar can be detected by incoherent scatter techniques. Therefore incoherent scatter observations of the plasma waves using UHF or VHF radars do not represent the whole spectrum of the Langmuir waves or plasma density oscillations excited in the interaction region of the HF wave.

Incoherent scatter radars can be used to investigate the natural plasma parameters such as electron density and temperature. In the absence of a modifying wave, weak incoherent backscatter signals are detected from Doppler shifted Langmuir and ion acoustic waves. In the case of ionospheric modification using a high power radio wave, Langmuir waves and the associated ion acoustic waves are strongly enhanced (Robinson, 1989). Such enhancements distort the incoherent scatter spectrum (Honary et al., 1999). As a result the ion line spectra cannot be analysed assuming a Maxwellian plasma distribution and therefore ionospheric parameters such as electron density and temperature cannot be reliably derived from the backscattered spectra. In addition, Langmuir turbulence persists when pumping the ionosphere on an electron gyroharmonic frequency (Honary et al., 1999). In order to estimate the electron temperature enhancements inside the heated volume, a theoretical model based on the analytical solution of the perturbation equations by Gurevich (1978) is used in this paper. Radar data above and below the ion-line enhancement region during Heater-on phases are contaminated by aliasing effects. 
Hence, the need for such modelling is not only because the ion-line spectra can not be analysed, but contamination in the radar data makes it impossible to estimate the electron temperature within the heated volume by extrapolating from above and below the interaction altitude.

\section{Experimental observations}

On 12 November 2001 the EISCAT HF facility (Rietveld et al., 1993) in Ramfjord, Norway, was operating at $5.423 \mathrm{MHz}$ with an effective radiative power (ERP) of $550 \mathrm{MW}$ and a beam width of $7^{\circ}$. The pump frequency corresponds to the fourth electron gyroharmonic at $215 \mathrm{~km}$, which is close to the HF interaction altitude. For the data analysed here the EISCAT radar beam was sweeping in $3^{\circ}$ steps moving from $3^{\circ} \mathrm{S}$ to $15^{\circ} \mathrm{S}$ of local zenith in a north-south meridian scan. The Heater pump beam direction was fixed at $9^{\circ} \mathrm{S}$ and was cycled with 2-min on, 2-min off. Artificial optical annuli, which were centred on the pump beam, formed a cylinder of optical emission above the pump wave reflection altitude and parallel to the magnetic field line direction. In $\sim 60$ s the annulus collapses into a blob while descending in altitude. The same descend can be observed in the EISCAT UHF backscatter power enhancements near the reflection altitude. For full details of the experimental setup refer to Kosch et al. (2004).

\section{Modelling}

A propagating electromagnetic wave can cause modification in the plasma and interacts with other propagating waves through a variety of non-linear processes (Robinson, 1989). This is the case when a high power HF radio wave heats the ionosphere during pumping experiments. Depending on conditions in the plasma, non-linearities can manifest themselves in two different characteristic types in the ionosphere. Firstly, for D-region up to lower F-region altitudes, the relatively high electron density and collision frequency causes thermal non-linearity to arise in the electrodynamic processes and the propagation of the electromagnetic wave. Secondly, in a collisionless plasma, where the electron mean free path is larger than the dimensions of the perturbing electric field associated with the modifying wave, transport processes such as thermal diffusion, thermal conductivity and particle diffusion are important. This is the case in the ionosphere above $\sim 200 \mathrm{~km}$ (Gurevich, 1978). Therefore two different mechanisms are responsible for electron heating in the ionosphere, one is the result of ohmic heating of the electrons due to collisions with the neutrals, and the other is known as anomalous heating and is caused by electromagnetic waves coupling to electrostatic waves (e.g. Langmuir and ion acoustic) in the plasma. The latter occurs only during O-mode heating of the iono- 
sphere.

Theoretical models discuss two different approaches known as cascading and cavitating or weak and strong Langmuir turbulence (WLT and SLT) to describe the non-linear processes of wave-plasma interaction in the F-region. Both of these processes (WLT and SLT) are responsible for the radar observations within the heated volume (Isham et al., 1999). Low latitude experiments at Arecibo show good agreement with the theoretical SLT spectra in which cavitating turbulence is formed by the growth and collapse of cavitons (DuBois et al., 1990), whereas the radar features observed for high latitude heating at Troms $\varnothing$ are mostly compatible with WLT, such as evidence of propagating Langmuir and ion acoustic waves in the radar plasma and ion line (Stubbe et al., 1992; Kohl et al., 1993). Furthermore, high latitude experiments do not confirm the existence of cavities and cavitons in the high and low frequency electrostatic perturbations, and parametric decay represents the major process responsible for Langmuir turbulence (Stubbe et al., 1992). However, when assuming either of the above approaches, the region of incoherent scatter, and associated ion line excitation, extends from the HF reflection height down to the altitude where Langmuir waves with large wave numbers are strongly Landau damped. Therefore Langmuir and ion acoustic waves cannot be generated outside this region (Stubbe et al., 1984). Extending down from the reflection level, the width of this region is $\sim 0.1 \mathrm{H}$, where $\mathrm{H}$ is the ionospheric scale height (Djuth et al., 1994).

Radar backscatter power measurements in the experiment analysed here show persistent ion line enhancements during heater-on cycles. The ion line enhancement echoes are a result of Langmuir turbulence near the reflection height $\left(z_{0}\right)$ and from the parametric decay of the pump beam into the Langmuir waves and ion acoustic waves at a lower altitude $\left(z_{a}\right) . z_{a}$ depends on the radar frequency $\left(f_{r}\right)$, ionospheric scale height $(\mathrm{H})$ and heating frequency $\left(f_{0}\right)$. Applying linear parametric instability theory to satisfy the Bragg condition in order to detect the plasma waves gives the following equation for the location of the Langmuir and ion waves (Stubbe et al., 1992):

$$
z_{a}=z_{0}-12 \frac{\kappa T_{e} f_{R}{ }^{2}}{m_{e} c^{2} f_{0}{ }^{2}} H
$$

where $m_{e}$ is electron mass, $T_{e}$ is electron temperature, $\kappa$ is Boltzmann's constant and $\mathrm{c}$ is the speed of light. In addition, ion acoustic waves connected to cascade processes can be detected at slightly higher altitudes $\left(z_{b}\right)$ where ion waves produce non-linear Landau damping cascades:

$$
z_{b}=z_{0}-3 \frac{\kappa T_{e} f_{R}{ }^{2}}{m_{e} c^{2} f_{0}{ }^{2}} H
$$

These echoes can contribute to the backscatter ion line enhancements in the vicinity 
of the O-mode reflection altitude (Djuth et al., 1994). Using a radar with sufficient range resolution one should detect a plasma and ion line at $z_{a}$ and ion line structure without a plasma line at $z_{b}$. Nevertheless, if the resolution of the radar is comparable to the separation of the excitation altitudes only one ion line enhancement will be observable (Stubbe et al., 1992). This is the case for the data analysed here. The EISCAT UHF radar data has a range resolution of $\sim 5.4 \mathrm{~km}$ which is similar to the estimated difference in height using Eq. 1 and $2(\sim 3 \mathrm{~km})$. Therefore the radar range resolution is not sufficient to resolve the two different signatures and we have used Eq. 2 to estimate the reflection height for each heater-on cycle. However, using Eq. 1 does not introduce significantly different results in calculating the temperature enhancements $(\leq 3 \%)$. No plasma line data were recorded on $12 / 11 / 2001$.

The effect of the electron temperature enhancements on the electron density depends on the altitude and ionospheric species. For instance, the effect of temperature dependent changes in the electron recombination rates are only important at E-region to lower F-region altitudes. At these altitudes electron heating affects the $\mathrm{O}_{2}^{+}$and $\mathrm{NO}^{+}$concentrations causing the electron density to increase. At altitudes where $\mathrm{O}^{+}$is the dominant species (above $\sim 200 \mathrm{~km}$, from the International Reference Ionosphere), diffusion is responsible for the plasma's response to the electron temperature enhancements, because the recombination rate coefficient for this species does not depend on the electron ambient temperature (Gurevich, 1978).

The stationary solutions to the linearised temperature $\left(T_{e}\right)$ and density $\left(N_{e}\right)$ perturbation equations (Gurevich, 1978) have been used to model the temperature enhancements inside the heated volume:

$$
\begin{aligned}
\triangle N= & \frac{N_{0} \gamma_{1}}{2} \frac{L_{N} L_{T} \tilde{Q}}{L_{N}^{2}-L_{T}^{2}}\left(\frac{\exp \left[-\left|x_{\|}-x_{0}\right| / L_{N}\right]}{L_{T}}-\frac{\exp \left[-\left|x_{\|}-x_{0}\right| / L_{T}\right]}{L_{N}}\right) \ldots \\
& -\frac{N_{0} k_{T}}{2} \frac{L_{N}^{2} \tilde{Q}}{L_{N}^{2}-L_{T}^{2}}\left(\frac{\exp \left[-\left|x_{\|}-x_{0}\right| / L_{T}\right]}{L_{T}}-\frac{\exp \left[-\left|x_{\|}-x_{0}\right| / L_{N}\right]}{L_{N}}\right) \\
\triangle T_{e}= & \frac{T_{e 0} \tilde{Q}}{2 L_{T}} \exp \left(-\frac{\left|x_{\|}-x_{0}\right|}{L_{T}}\right)
\end{aligned}
$$

Ion heating is assumed to be negligible. The unperturbed electron density $\left(N_{0}\right)$ and temperature $\left(T_{e 0}\right)$ are derived from EISCAT measurements prior to each heater cycle. $x_{0}$ is the reflection altitude of the pump wave, $x_{\|}$is the coordinate along the magnetic field line direction. It has been assumed that the dimensions of electron temperature and density inhomogeneity perpendicular to the magnetic field is larger than the electron mean free path so that only longitudinal thermal conductivity and diffusion become important. $L_{T}$ and $L_{N}$ are the characteristic length of temperature and density inhomogeneity. $k_{T}$ is the thermal-diffusion ratio $k_{T}=D_{T e a} / D_{a}$, in which $D_{a}$ is the ambipolar diffusion coefficient, $D_{T e a}$ is electron coefficient of ambipolar diffusion, $\gamma_{1}$ is the ionisation equilibrium shift coefficient, $\gamma_{1}=\frac{T_{e 0}\left(\partial q_{r} / \partial T_{e}\right)}{N_{0}\left(\partial q_{r} / \partial N\right)}$, 
in which $q_{r}$ is electron loss per $\mathrm{cm}^{3}$ per second as a result of recombination.

The height integrated heat input per unit volume per second is given by $\tilde{Q}=\int \varphi E_{0}^{2} / E_{P}^{2}$ where $\varphi=\varphi_{P} / \varphi_{T}, E_{0}$ is the modifying electric field, $E_{P}$ is the ambient electric field of the plasma, $\varphi_{P}$ is the polarisation factor and $\varphi_{T}$ is the non-isothermy factor. Using Eq. (3) the heat input of the electromagnetic wave can be calculated. Knowing the heat input per unit volume, one can derive from Eq. (4) the temperature enhancements associated with the heating at the height indicated by the ion line enhancement altitude.

Neutral atmosphere parameters such as the density of neutral species have been obtained from the MSIS-90 atmospheric model. It has been argued that MSIS does not agree well with the observations at high latitudes. The MSIS model is based on extensive data input from different sources such as satellites and incoherent scatter radars. At high latitudes and for F-region altitudes the data coverage is relatively poor and therefore it is mostly influenced by the mid-latitude measurements (E. Griffin, private communication). However, comparisons between FPI measured neutral temperatures and those from the MSIS model show good agreement for quiet geomagnetic activity conditions (Kosch et al., 2000a). In our case, $\mathrm{Kp}=0$ throughout the experiment. Therefore we use MSIS model with high confidence for the neutral atmosphere. The ion composition and densities have been derived from the International Reference Ionosphere (IRI-2001). The same comments regarding reliability apply to this model.

Figure 1 summarises the electron temperature modelling results. The top panel shows the logarithm of raw backscattered power from the EISCAT UHF radar. The intervals indicated by black lines represent heater-on cycles. The labels are radar zenith angles south of vertical. Persistent and strong ion line enhancements are mostly observed in the direction of magnetic field line $\left(12^{\circ} \mathrm{S}\right)$ and $9^{\circ} \mathrm{S}$. The backscatter coming from $6^{\circ} \mathrm{S}$ or $15^{\circ} \mathrm{S}$ do not show persistent enhancements during heating. For $3^{\circ} \mathrm{S}$ no useful backscatter was obtained. For the cases where the ion line enhancements do not persist throughout the heater-on cycle, an extrapolation has been performed to derive the height of ion line enhancements after 2 minutes. By fitting a second order polynomial to the ion line enhancements, the final height has been estimated for these cases only.

Knowing the start and end height of the backscattered enhancements, and calculating the ionospheric scale height $(\mathrm{H})$ from the EISCAT electron density profile, the HF reflection height has been computed. The middle panel of Fig. 1 shows the calculated HF reflection heights corresponding to the start and end of the heateron cycles (blue and red curve, respectively) derived from Eq. (2). The black curve is the reflection altitude calculated from EISCAT electron density profile. This is generally within 1-2 range gates of HF reflection altitude predicted by Eq. 2. The clear descent in the height of the backscatter enhancements represents the change in electron density during each heater-on cycle because the pump frequency remains 
constant. Therefore, for each heater cycle, the changes in the electron density of the interaction altitude is equal to the difference of cold plasma electron density at the start and end altitude. Based on this assumption and using Eqs. (3) and (4) the final temperature has been calculated using the EISCAT electron temperature measurements just before pump-on at the starting altitude $\left(T_{e 0}\right)$.

The bottom panel of Fig. 1 shows the initial electron temperature (blue curve) and density (green curve) from EISCAT data and the modelled final electron temperature (red curve) all for the final altitude of each heater-on cycle. The error bars stem from the radar measurement uncertainty of initial density and temperature. The average modelled temperature uncertainty is $\sim 11 \%$. There are no clear ion line enhancements for the periods when the heater was pointing $3^{\circ} \mathrm{S}$, therefore it is not possible to derive the electron temperature for these intervals. It is important to emphasize that the estimated temperature, has been calculated for one single altitude (end height of the ion line enhancement) and after 2-minutes of each heating period (steady state).

\section{Discussion}

For our experiment the average temperature enhancement for all directions is $\sim 4800$ $\mathrm{K}$. Table 1 shows the average electron temperature for different radar zenith angles. The maximum temperature $(5700 \mathrm{~K})$ occurs for $9^{\circ} \mathrm{S}$ parallel to the pump beam direction. Although the pump power is approximately half of maximum in the magnetic field line direction $\left(12^{\circ} \mathrm{S}\right)$, the electron temperature increases up to $5500 \mathrm{~K}$ in the magnetic zenith. This is consistent with Rietveld et al. (2003) who found the temperature maximum at $12^{\circ} \mathrm{S}$. Their angular scan resolution was only $6^{\circ}$ and no measurements were made at $9^{\circ} \mathrm{S}$. For the same angular step, the modelled temperature drops off more rapidly towards local zenith $\left(2700 \mathrm{~K}\right.$ at $\left.6^{\circ} \mathrm{S}\right)$ than beyond the magnetic zenith $\left(5100 \mathrm{~K}\right.$ at $\left.15^{\circ} \mathrm{S}\right)$.

Temperatures up to $\sim 3000 \mathrm{~K}$ have been measured by Rietveld et al. (2003) using an ERP of $\sim 205 \mathrm{MW}$. In the region of the F-layer maximum where the electron-ion collision frequency is much higher than the electron-neutral collision frequency, the electronic thermal conductivity coefficient $\left(\kappa_{e}\right)$ increases rapidly with electron temperature: $\kappa_{e} \sim T_{e}^{5 / 2}$ (Gurevich, 1978; Schunk and Nagy, 1978). Hence, doubling the ERP does not double the electron temperature. Our result is consistent with that of Rietveld et al. (2003): (i.e. $\left.\left(\frac{550 M W}{205 M W}\right) \sim\left(\frac{4800 K}{3000 K}\right)^{2 / 5}\right)$.

The electron density at the reflection altitude is given by $f=8.98 \sqrt{N}$. For $\mathrm{f}=5.423 \mathrm{MHz}, N_{e}=3.6 \times 10^{11} \mathrm{~m}^{-3}$. Assuming the average heated bulk electron temperature of $4800 \mathrm{~K}$ has a Maxwellian distribution, the characteristic kinetic energy of the electrons is $\sim 0.31 \mathrm{eV}$. The excitation of the 557.7 and $630.0 \mathrm{~nm}$ emissions require electrons with energies higher than $4.17 \mathrm{eV}$ and $1.96 \mathrm{eV}$, re- 
spectively (Rees, 1989). Therefore the flux of the electrons producing these two emissions can be calculated from the Maxwellian distribution. About $4 \%$ of the energetic electrons contribute to the production of photons by collisions (Rees, 1963). Integrating $4 \%$ of the total electron population greater than $1.96 \mathrm{eV}(4.17 \mathrm{eV})$ a total of $1.87 \times 10^{8} \mathrm{~m}^{-3}\left(2.96 \times 10^{5} \mathrm{~m}^{-3}\right)$ electrons contribute to creating the $630.0 \mathrm{~nm}$ $(557.7 \mathrm{~nm})$ emission. Considering a uniform spherical emission volume of radius $13.5 \mathrm{~km}$ at $\sim 220 \mathrm{~km}$ altitude $(-3 \mathrm{~dB}$ beam size $)$, the estimated photon production rate is $\sim 27$ Rayleighs $(\mathrm{R})$ for $630.0 \mathrm{~nm}$ and $\sim 0.05 \mathrm{R}$ for $557.7 \mathrm{~nm}$. These intensities are significantly smaller than those observed by Kosch et al. (2004) (300 R for red line and $100 \mathrm{R}$ for green line). This indicates that the electron flux required to explain the optical intensities detected are much higher than the Maxwellian flux. Hence, the assumption of a thermal distribution for the accelerated electrons cannot be applied to our observations. This is in agreement with the persistent ion line enhancements in the backscatter spectra within the heated volume which indicate a turbulent plasma.

It is important to note that the observed electron density changes cannot be due to further ionisation of species such as $\mathrm{O}^{+}, \mathrm{O}_{2}^{+}$and $\mathrm{N}_{2}^{+}$. This is due to the fact that the ionisation threshold for such ions are $\mathrm{O}^{+}: 13.6 \mathrm{eV}, \mathrm{O}_{2}^{+}: 12 \mathrm{eV}$ and $\mathrm{N}_{2}^{+}: 15.5$ $\mathrm{eV}$ (Rees, 1989) which is $\gg 0.31 \mathrm{eV}$, which corresponds to the average modelled temperature of $4800 \mathrm{~K}$. Therefore the heated bulk plasma temperature is not high enough to ionise these species.

Modelling studies of the artificial optical emissions at $630.0 \mathrm{~nm}$ show that the observed emissions could be caused by electrons with very high energy ( $\gtrsim 5000 \mathrm{~K}$ ) inside small scale striations produced by the electromagnetic pump waves (Gurevich and Milikh, 1997). Gurevich et al. (1995) suggested that the striations, or magnetic field aligned irregularities, are regions with very high electron temperature which are within larger volumes of ambient plasma with a lower temperature. However, doubt has been cast on this theory because it is inconsistent with the pressure balance along the magnetic field (Borisov et al., 2004). The incoherent scatter radar is only capable of measuring the electron temperature assuming a uniform thermal plasma in the scattering volume. Hence, the temperatures measurements from radar data in such circumstances would be the weighted average of plasma temperatures within the radar beam.

\section{Conclusion}

The model predicts an average electron temperature of $\sim 5500 \mathrm{~K}$ along the magnetic field line inside the heated volume. The maximum temperature of $\sim 5700 \mathrm{~K}$ occurs at $9^{\circ} \mathrm{S}$, i.e., where the pump power is maximum. Neither the red nor green line steady-state optical emission can be explained by a thermal plasma of 4800 $\mathrm{K}$. Clearly, non-linear electron acceleration is active throughout the experiment. 
Table 1. Average temperature from different zenith angles

\begin{tabular}{cc}
\hline $\begin{array}{c}\text { Radar zenith } \\
\text { angle }\left(^{\circ}\right)\end{array}$ & $\begin{array}{c}\text { Average temperature } \\
\text { in heated volume }(\mathrm{K})\end{array}$ \\
\hline $6^{\circ}$ & 2700 \\
$9^{\circ}$ & 5700 \\
$12^{\circ}$ & 5500 \\
$15^{\circ}$ & 5100 \\
\hline
\end{tabular}

The radar ion line enhancements indicate that Langmuir turbulence is an important mechanism for electron acceleration.

\section{Acknowledgements}

The authors thank Paul Gallop for filtering the EISCAT radar data. EISCAT is an international scientific association supported and operated by the research organisations of Finland, France, Germany, Japan, Norway, Sweden and the United Kingdom.

\section{References}

Borisov, N., Senior, A., Honary, F., 2004. Stationary state and relaxation of artificial irregularities excited in ionospheric heating experiments. To appear in J. Plasma Phys.

Djuth, F. T., Stubbe, P., Sulzer, M. P., Kohl, H., Rietveld, M. T., Elder, J. H., 1994. Altitude characteristics of plasma turbulence excited with the Troms $\varnothing$ superheater. J. Geophys. Res. 99 (A1), 333-339.

DuBois, D. F., Rose, H. A., Russell, D., 1990. Excitation of strong Langmuir turbulence in plasmas near critical density: Application to HF heating of the ionosphere. J. Geophys. Res. 95 (A5), 21221-21272.

Gordon, W. E., Carlson, H. C., 1974. Arecibo heating experiments. Radio Sci. 9, 1041-1047.

Gordon, W. E., Carlson, H. C., Showen, R. L., 1971. Ionospheric heating at Arecibo: First tests. J. Geophys. Res. 76, 7808-7813.

Gurevich, A. V., 1978. Nonlinear phenomena in the ionosphere. Elsevier, NorthHolland.

Gurevich, A. V., Lukyanov, A. V., Zybin, K. P., 1995. Stationary state of isolated striations developed during ionospheric modification. Physics Letters A 206 (A1), 247-259.

Gurevich, A. V., Milikh, G. M., 1997. Artificial airglow due to modifications of the ionosphere by powerful radio waves. J. Geophys. Res. 102 (A1), 389-394.

Honary, F., Robinson, T. R., Wright, D. M., Stocker, A. J., Rietveld, M. T., McCrea, 
I., 1999. First direct observations of the reduced striations at pump frequencies close to the electron gyroharmonics. Ann. Geophys. 17 (A4), 1235-1238.

Honary, F., Stocker, A. J., Robinson, T. R., Jones, T. B., Wade, N. M., Stubbe, P., Kopka, H., 1993. EISCAT observations of electron temperature oscillations due to the action of high power hf radio waves. J. Atmos. Terr. Phys. 55 (10), 1433-1448.

Isham, B., Hoz, C. L., Rietveld, M. T., Hagfors, T., Leyser, T. B., 1999. Cavitating Langmuir turbulence observed during EISCAT high latitude ionospheric interaction experiments. Phys. Rev. Lett. 83 (13), 2576-2579.

Jones, T. B., Robinson, T., , Stubbe, P., Kopka, H., 1986. EISCAT observations of heated ionosphere. J. Atmos. Terr. Phys. 48 (A1), 1027-1035.

Jones, T. B., Robinson, T., Kopka, H., Stubbe, P., 1982. Phase changes induced in a diagnostic radio wave passing through a heated region of the aurora ionosphere. J. Geophys. Res. 87 (A3), 1557-1564.

Kohl, H., Kopka, H., Stubbe, P., Rietveld, M., 1993. Introduction to ionospheric heating experiments at Troms $\varnothing$ Part 2: Scientific problems. J. Atmos. Terr. Phys. 55 (4/5), 601-613.

Kosch, M. J., Ishii, M., Kohsiek, A., Rees, D., Schlegel, K., Hagfors, T., Cierpka, K., 2000a. A comparison of thermospheric winds and temperatures from FabryPerot interferometer and EISCAT radar measurements with models. Adv. Space Res. 26 (6), 979-984.

Kosch, M. J., Rietveld, M. T., Hagfors, T., Leyser, T. B., 2000b. High-latitude HFinduced airglow displaced equatorwards of the pump beam. Geophys. Res. Lett. 27, 2817-2820.

Kosch, M. J., Rietveld, M. T., Senior, A., McCrea, I., Kavanagh, A. J., Isham, B., Honary, F., 2004. Novel artificial optical annular structures in the high latitude ionosphere over EISCAT. Geophys. Res. Lett. 31, 1235-1238.

Mingaleva, G. I., Mingalev, V., 1997. Response of the convecting high-latitude F layer to a powerful HF wave. Ann. Geophys. 15 (10), 1291-1300.

Rees, M. H., 1963. Auroral ionisation and excitation by incident energetic electrons. Planet. Space Sci. 11 (A1), 1209-1218.

Rees, M. H., 1989. Physics and Chemistry of the Upper Atmosphere. Cambridge University Press.

Rietveld, M. T., Kohl, H., Kopka, H., Stubbe, P., 1993. Introduction to ionospheric heating at Troms $\varnothing-1$. Experimental overview. J. Atmos. Terr. Phys. 55, 577-599.

Rietveld, M. T., Kosch, M. J., Blagoveshchenskaya, N. F., Kornienko, V. A., Leyser, T. B., Yeoman, T. K., 2003. Ionospheric electron heating, optical emissions, and striations induced by powerful HF radio waves at high latitudes: Aspect angle dependence. J. Geophys. Res. 108, 2/1-2/16, doi:10.1029/2002JA009543.

Robinson, T. R., 1989. The heating of the high latitude ionosphere by high power radio waves. Physics Reports 179 (2/3), 79-209.

Schunk, R. W., Nagy, A. F., 1978. Electron temperatures in the F region of the ionosphere: theory and observations. Rev. of Geophys. Space Phys. 16 (3), 355399.

Sergienko, T., Gustavsson, B., Å. Steen, Brändström, U., Rietveld, M., Leyser, 
T. B., Honary, F., 2000. Analysis of excitation of the $630.0 \mathrm{~nm}$ airglow during a heating experiment in Troms $\varnothing$ on February 16, 1999. Phys. Chem. Earth (B) $25(5-6), 531-535$.

Stocker, A. J., Honary, F., Robinson, T. R., Jones, T. B., Stubbe, P., Kopka, H., 1992. EISCAT observations of large scale electron temperature and electron density perturbations caused by high power HF radio waves. J. Atmos. Terr. Phys. 54 (11/12), 1555-1572.

Stubbe, P., Kohl, H., Rietveld, M. T., 1992. Langmuir turbulence and ionospheric modification. J. Geophys. Res. 97 (A5), 6285-6297.

Stubbe, P., Kopka, H., Thidé, B., Derblom, H., 1984. Stimulated electromagnetic emission: A new technique to study the parametric decay instability in the ionosphere. J. Geophys. Res. 89 (A9), 7523-7536.

Vas'kov, V. V., Dimant, Y. S., Ryabova, N. A., 1993. Magnetospheric plasma thermal perturbations induced by resonant heating of the ionospheric F-region by high-power radio wave. Adv. Space Res. 13 (10), 25-33. 

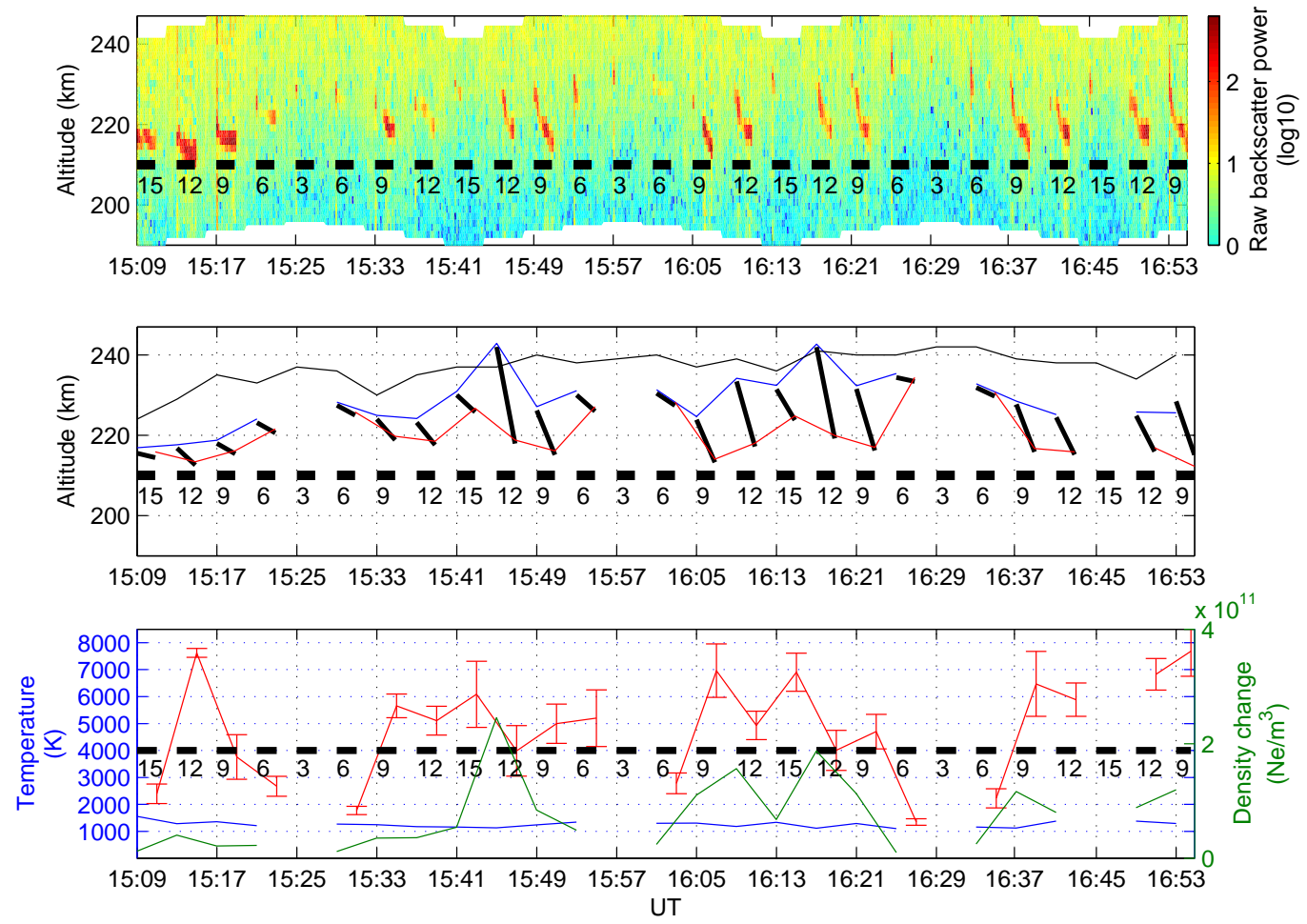

Fig. 1. Electron temperature modelling results. The top panel shows the logarithm of raw backscattered power from the EISCAT UHF radar. The middle panel shows the calculated HF reflection heights corresponding to the start and end of the heater-on cycles (blue and red curve, respectively) and the reflection altitude calculated from EISCAT electron density profile (black curve). The bottom panel shows the initial electron temperature (blue curve) and density (green curve) from EISCAT data and the modelled final electron temperature (red curve) all for the final altitude of each heater-on cycle. The error bars stem from the radar measurement uncertainty of initial density and temperature. 